\title{
Optimal Customized Bundle Pricing
}

\author{
Ameera Ibrahim
}

\begin{abstract}
We study the pricing of customized bundles, where a firm sets a bundle price that depends on the size of the bundle, not on its individual products. With the objective of maximizing the firm's profit, we formulate a nonlinear mixed-integer program based on a prior model from the literature. A linear mixed-integer formulation is developed which effectively enables an exact solution to the problem.
\end{abstract}

Index Terms-Bundling, mixed-integer programming, pricing.

\section{INTRODUCTION}

Consider a multi-product firm having customers that are willing to buy one or more of its products. The firm faces the problem of pricing the products so that the overall profit is maximized. An example of such firm can be a software company with many modules that can be bundled in a software package, a cable television company, a digital music retailer or any other firm selling low marginal cost products.

To solve this pricing problem, firms can choose from several pricing schemes. Some schemes are simple such as component pricing (CP) where products are priced individually, and pure bundling (PB) where only one bundle that includes all the products is offered and priced. Others are more complex, like mixed bundling (MB) which is theoretically the pricing scheme that guarantees the best pricing policy. $\mathrm{MB}$ investigates all the possible combinations of product bundles and chooses the optimal assortment of bundles that increases the profit [1]. This technique considers $2^{J}-1$ possible bundle prices for $J$ products, and thus is complex to solve and to implement since it results in a large number of pricing options. That gives the motivation to study other bundle pricing techniques that offer less number of alternative prices.

Customized bundle pricing (CBP) (also known in the literature as cardinality bundling or bundle-size pricing), involves setting one price for a given-size bundle, so that bundle price depends on the number of products in the bundle, not on the specific products included.

For example, consider a firm selling three different products, CBP sets one price for the purchase of any single product, a second price for the purchase of any two products, and a third price for purchasing all three. Indeed, the optimal solution might choose not to offer all three bundles. It could be more profitable to offer two of them for instance, or even one. Thus, in addition to setting the optimal price for a bundle, the CBP approach finds the optimal assortment of bundles to

Manuscript received February 7, 2019; revised May, 2, 2019.

Ameera Ibrahim is with the Business Analytics Department, School of Economics and Business Administration, Saint Mary's College of California, United States of America (e-mail: ai7@stmarys-ca.edu). offer in order to maximize the firm's profit while increasing consumer welfare.

Bundle pricing in general has been extensively studied in the literature. One of the earliest studies on bundling is that by Stigler in [2]. His model sets the basis over which most bundling studies are built on. The model captures customer demand through a vector of reservation prices for products, where customers choose the product that maximizes their surplus (difference between reservation price and product price). References [3], [4] build on the Stigler model and highlight the profitability of the mixed bundling approach. Reference [5] uses the Law of Large Numbers to show that bundling a large number of low cost information products can be profitable. Following them, many studies on bundle pricing evolved for different demand circumstances and various applications. The work in [6] for example, shows the benefits of $\mathrm{MB}$ compared to $\mathrm{CP}$ and $\mathrm{PB}$ as the authors formulate a nonlinear mixed-integer program to solve a pricing application in the wireless telecommunications industry.

Although work in the customized bundle pricing area is relatively sparse, prior work has suggested that this bundling approach has attractive features when pricing low marginal cost products. Most relevant to our work is that in [7] where the authors formulate the CBP problem as a nonlinear mixed-integer program, then due to its non-convex nature, use Lagrangian relaxation and heuristics to derive bounds. Reference [8] analyzes the CBP mechanism analytically and identify circumstances under which it becomes particularly attractive than other pricing mechanisms. In [9] the authors use real data to argue that the profit from CBP is nearly the same as that of $\mathrm{MB}$ and is almost certainly more profitable than either $\mathrm{CP}$ or $\mathrm{PB}$. One of the most recent works on CBP is that in [10] where the authors study both the discrete and continuous versions of the problem. They reformulate the discrete CBP model from [8] into a linear program that allows an efficient solution procedure.

In this paper we first consider the model in [7] and extend it to include the notion of customer segments instead of individual customers. We reformulate the nonlinear model into a linear mixed-integer program where an exact solution to the CBP problem is obtained.

This paper is organized as follows: In section II, we present a definition of the problem and its notations, formulate the original nonlinear mixed-integer model, and present the proposed linear mixed-integer formulation along with an example. A computational analysis is carried in section III for different size instances to demonstrate the efficiency of the proposed model. Section IV concludes with a discussion of our major findings and avenues for future research. 


\section{PROBLEM FORMULATION AND SOLUTION APPROACH}

\section{A. Mixed-Integer Nonlinear Formulation}

The customized bundle pricing problem models a situation where a firm offers its customers a variety of products, and customers may choose a bundle out of any of those products as long as the number of products in the bundle matches the bundle size that the firm announced to offer for a given price. Our basic model extends the main model in [7] as we add the notion of customer segments and their sizes. Customers are grouped into $I$ customer segments based on similar purchase behavior characteristics. The firm offers a variety of $J$ low marginal cost products. Our model is developed from the firm's perspective seeking to decide which bundles to offer and how to price them so that it maximizes the profit while consumer welfare is also maximized.

One of the general assumptions of this setting involves that demand is captured through reservation prices of the products which estimates the maximum price this customer segment is willing to pay for each bundle size.

The following notation is introduced to model the problem: Input Parameters

- $I$ : There are $I$ customer segments in the market.

- $J$ : The firm offers a variety of $J$ products.

- $i$ : Index for customer segments, where $i \in[1, \ldots, I]$.

- $j$ : Index for products, where $j \in[1, \ldots, J]$.

- $S_{i}$ : The size of customer segment $i$.

- $M$ : Marginal menu cost of adding one more bundle choice to the menu.

- $\quad C_{j}$ : The cost of creating a bundle of $j$ products.

- $\quad R_{i j}$ : Total reservation price of customer segment $i$ 's top $j$ favorite products.

Decision Variables

- $\quad P_{j}$ : The price of a bundle of $j$ products. A price of 0 implies that the firm is not offering, thus not pricing, bundle $j$.

- $\quad X_{i j}$ : A binary variable that is 1 if customer segment $i$ chooses to buy a bundle of $j$ products, and is 0 otherwise.

- $Y_{j}$ : A binary variable that is 1 if the bundle of $j$ products is offered in the menu, and is 0 otherwise.

The problem is then to determine the optimal assortment of bundles and their optimal prices. The following formulation is a non-linear mixed-integer program for solving the customized bundle pricing problem, denoted by CBPP.

CBPP: Maximize

$$
\sum_{i=1}^{I} \sum_{j=1}^{J}\left(P_{j}-C_{j}\right) X_{i j} S_{i}-\sum_{j=1}^{J} M Y_{j}
$$

Subject to

$$
\begin{gathered}
\sum_{k=1}^{J}\left(R_{i k}-P_{k}\right) X_{i k} \geq\left(R_{i j}-P_{j}\right) Y_{j}, \quad \forall i, j \\
\left(R_{i j}-P_{j}\right) X_{i j} \geq 0, \quad \forall i, j \\
\sum_{j=1}^{J} X_{i j} \leq 1, \quad \forall i \\
X_{i j} \leq Y_{j}, \quad \forall i, j
\end{gathered}
$$

$$
P_{j} \leq \max _{i \in[I]}\left\{R_{i j}\right\} Y_{j}, \quad \forall j
$$

$$
X, Y \in\{0,1\}, P \geq 0 \text {. }
$$

The objective function (1) maximizes the firm's total profit, which is the profit obtained from each customer segment's purchase minus the cost of adding the chosen bundle(s) to the menu. Constraints (2) define consumer surplus as the difference between the customer segment's reservation price of the bundle and the market price, and ensure that each customer segment chooses a bundle that maximizes its surplus (incentive compatibility constraints). Constraints (3) ensure that the customer segment chooses a bundle only if its price is not higher than the reservation price of this bundle. Constraints (4) ensure that each customer segment can purchase at most one bundle. Constraints (5) ensure that a customer segment can purchase a bundle only if it is offered by the firm. Constraints (6) enforce upper bounds on prices based on the highest reservation price across all customer segments. In addition, these constraints logically relate the pricing and the assortment variables so that bundles that are not offered are not priced. Constraints (7) enforce binary restrictions for both consumer purchases and bundle offerings decisions, and non-negativity constraints for bundle prices decisions.

Model CBPP is clearly nonlinear as shown in (1), (2) and (3) through the two nonlinear terms: $P_{j} X_{i j}$ and $P_{j} Y_{j}$. In order to solve CBPP, a mixed-integer nonlinear program (MINLP) solver can be used. We use BARON to solve the problem to optimality. BARON guarantees termination with a global optimal solution. It can find a solution to small size problems in seconds. However, real-life instances of medium and large sizes will require the solver to run for extensive amounts of time before converging and thus, it is not practical to use.

Before we proceed to our efficient approach for solving the problem, we demonstrate the current non-linear model using an example.

Example 1. Consider a scenario with $I=3$ customer segments having 10 customers each, thus $S_{i}=10$ for all $i$. There are 4 products and thus $J=4$. Marginal menu cost $M=$ 10 and bundle costs $C_{j}=0$ for all $j$. The reservation prices of customer segments for each bundle size are given in Table I.

TABLE I: RESERVATION PRICES FOR EXAMPLE 1

\begin{tabular}{llll}
\hline \hline Bundle Size & $\mathrm{I}_{1}$ & $\mathrm{I}_{2}$ & $\mathrm{I}_{3}$ \\
\hline 1 & 16 & 36 & 40 \\
2 & 30 & 50 & 56 \\
3 & 45 & 66 & 85 \\
4 & 51 & 80 & 100 \\
\hline \hline
\end{tabular}

The optimal solution is found in 0.2 seconds using the solver BARON under AMPL. Bundles 3 and 4 are offered with $P_{3}=45$ and $P_{4}=59$. Customer segments 1,2 , and 3 buy bundles 3, 4 and 4 respectively. The optimal profit is 1610 .

Note that in this example, the optimal assortment of bundles is composed of only two bundles out of four possible bundle options. Pricing schemes with less number of pricing options are more attractive to the industry in general due to its ease of implementation.

A pure bundling strategy for this problem will offer only one bundle of all the products. For the data in Example 1, the PB 
approach finds an optimal profit of 1590. The optimal solution in that case offers bundle 4 with $\mathrm{P}_{4}=80$. Customer segments 2 and 3 buy bundle 4 , while customer segment 1 is excluded from the market since its reservation price is less than the market price.

\section{B. Mixed-Integer Linear Formulation}

In this subsection, a linear formulation of the problem is proposed which allows an efficient solution for real-life size instances.

The computational intractability of Model CBPP can be greatly alleviated by developing an equivalent mixed-integer linear reformulation. To this end, the following proposition shows that the nonlinear term $P_{j} Y_{j}$ can be replaced by simply $P_{j}$ :

Proposition 1. It is valid to substitute $P_{j} Y_{j} \equiv P_{j}$ in model CBPP.

Proof.

- If $Y_{j}=0$, then $P_{j} Y_{j}=0$ and $P_{j}=0$ by Constraint (6), and thus $P_{j} Y_{j}=P_{j}$.

- If $Y_{j}=1$, then $P_{j} Y_{j}=P_{j}$. $\square$

The result established in Proposition 1 is intuitive in that a bundle of $j$ products that is not selected in the assortment will not be priced by the firm. Note, however, that a similar result does not necessarily hold for $P_{j} X_{i j}$, i.e., $X_{i j}=0$ does not necessarily imply that $P_{j}=0$. In fact, a bundle that is not selected by one customer segment $i$ can be purchased by another segment and ought to be priced by the retailer. Thus, we introduce a new variable $G_{i j}=P_{j} X_{i j}$.

The results from Proposition 1 and the introduction of the auxiliary nonnegative continuous variable $G_{i j}$, in lieu of the nonlinear term $P_{j} X_{i j}$ along with the linearizing constraints in (13) and (14), enable the following linear reformulation of CBPP, denoted by LCBPP.

\section{LCBPP: Maximize}

$$
\begin{gathered}
\sum_{i=1}^{I} \sum_{j=1}^{J}\left(G_{i j}-C_{j} X_{i j}\right) S_{i}-\sum_{j=1}^{J} M Y_{j} \\
\sum_{k=1}^{J}\left(R_{i k} X_{i k}-G_{i k}\right) \geq R_{i j} Y_{j}-P_{j}, \quad \forall i, j \\
R_{i j} X_{i j}-G_{i j} \geq 0, \quad \forall i, j \\
\sum_{j=1}^{J} X_{i j} \leq 1, \quad \forall i \\
X_{i j} \leq Y_{j}, \quad \forall i, j \\
P_{j} \leq \max _{i \in[I]}\left\{R_{i j}\right\} Y_{j}, \quad \forall j \\
G_{i j} \geq P_{j}-\max _{k \in[I]}\left\{R_{k j}\right\}\left(1-X_{i j}\right), \quad \forall i, j \\
G_{i j} \leq P_{j}, \quad \forall i, j \\
X, Y \text { Binary, } P \geq 0 .
\end{gathered}
$$

For completeness, the following proposition establishes the validity of LCBPP:

Proposition 2. Model LCBPP is a valid reformulation of Model CBPP.

Proof.

Consider the substitution relationships $G_{i j} \equiv P_{j} X_{i j}$, for all $i$ and $j$, and note that:
- If $X_{i j}=0$, then $P_{j} X_{i j}=0$ and we need to verify that $G_{i j}$ $=0$. From (9) and the non-negativity restriction on the $G$-variables in (15), we have that $G_{i j}=0$. Under this condition, constraints (13) and (14) hold true, and $G_{i j}=P_{j} X_{i j}$.

- If $X_{i j}=1$, we need to verify that $G_{i j}=P_{j}$, which is jointly enforced by constraints (13) and (14). $\square$

Constraints (13) above impose a lower bound to the new variable $G_{i j}$ using reservation prices and conditioned with whether the bundle was selected or not. Constraints (14) limit $G_{i j}$ to take at most the value of bundle $j$ 's price.

We use LCBPP to solve the scenario in Example 1 above. Using the linear solver Gurobi, the optimal solution is found in 0.14 seconds. The power of our exact solution approach can be demonstrated by solving large instances that the nonlinear model was unable to solve in a reasonable amount of time. Thus next, we solve medium and large size instances to demonstrate the efficiency of LCBPP.

\section{Computational AnAlysis AND Results}

In this section, two computational experiments are discussed. The first demonstrates the efficiency of the linear formulation approach, and the second compares it to an existing bundle pricing approach from the literature.

In the first experiment, the linear model LCBPP is solved for different data instances. We measure the average time it took the computer to solve the problem to optimality as we increase the number of customer segments $I$ and the number of offered products $J$. This captures the performance limits of the proposed model. The average number of bundles selected by the model will also be displayed with each run. For each observation, three different instances of the same size are solved and averaged. Table II shows results of the numerical analysis.

The given parameters of the model are generated randomly within certain predetermined data ranges. Solutions are obtained using the solver Gurobi under AMPL. All runs took place on a PC with Intel(R) Core(TM) i7-4790 CPU, 3.60 $\mathrm{GHz}$, having $16 \mathrm{~GB}$ of RAM and running Windows 7 operating system.

TABLE II: COMPUTATIONAL ANALYSIS OF LCBPP

\begin{tabular}{llll}
\hline \hline Observation & Size $(I, J)$ & $\begin{array}{l}\text { Avg. no. } \\
\text { of bundles }\end{array}$ & $\begin{array}{l}\text { Avg. CPU } \\
\text { time (sec) }\end{array}$ \\
\hline 1 & $(5,10)$ & 3 & 0.03 \\
2 & $(5,50)$ & 2 & 0.21 \\
3 & $(5,100)$ & 2 & 0.32 \\
4 & $(5,500)$ & 2 & 6.90 \\
5 & $(10,10)$ & 3 & 0.23 \\
6 & $(10,50)$ & 4 & 1.12 \\
7 & $(10,100)$ & 3 & 4.32 \\
8 & $(10,500)$ & 2 & 32.87 \\
\hline \hline
\end{tabular}

As shown in Table II, on average, problems of up to 500 products can be solved to optimality in seconds. An interesting behavior of the model is that the average number of bundles does not necessarily increase with the increase in the number of products. For example, Table II shows that a firm with 500 products will offer two bundle sizes only. This behavior could be of much interest to firms that are limited with a small number of price offerings for their products. 
In the second experiment, we compare the performance of our customized bundle pricing approach against pure bundling. We solve the same instances of the first experiment using the pure bundling approach and compare profits as shown in Table III.

TABLE III: CUSTOMIZED BundLE PRICING COMPARED TO PURE BUNDLING

\begin{tabular}{lllll}
\hline \hline Observation & Size $(I, J)$ & $\begin{array}{l}\text { PB Avg. } \\
\text { Profit }\end{array}$ & $\begin{array}{l}\text { CBP Avg. } \\
\text { Profit }\end{array}$ & $\begin{array}{l}\text { Avg. Profit } \% \\
\text { Increase }\end{array}$ \\
\hline 1 & $(5,10)$ & 19392 & 21864 & $12.7 \%$ \\
2 & $(5,50)$ & 126226 & 128472 & $1.8 \%$ \\
3 & $(5,100)$ & 179587 & 180653 & $0.6 \%$ \\
4 & $(5,500)$ & 1222513 & 1228251 & $0.5 \%$ \\
5 & $(10,10)$ & 54700 & 59527 & $8.8 \%$ \\
6 & $(10,50)$ & 239149 & 243667 & $1.9 \%$ \\
7 & $(10,100)$ & 460849 & 472340 & $2.5 \%$ \\
8 & $(10,500)$ & 2379698 & 2384250 & $0.2 \%$ \\
\hline \hline
\end{tabular}

Table III shows that under the same circumstances, our proposed customized bundle pricing approach offers better assortments, and thus better average profits, than pure bundling. The overall average profit increase for all instances in our experiment is $3.6 \%$.

\section{DISCUSSION AND CONCLUSIONS}

Customized bundle pricing is a flexible pricing scheme adopted by the industry due to its practicality in offering a small number of pricing options. Yet, that scheme is not widely studied in the literature. This paper provides an exact solution approach to the customized bundle pricing problem. A linear mixed-integer program that solves the problem to optimality is successfully developed and formulated. Due to the complexity of the original nonlinear mixed-integer formulation, previous literature reverted to approximation methods and heuristics to solve the problem. To the best of our knowledge, our formulation is the first to utilize linearization techniques to obtain a linear formulation of the problem. Our numerical computations demonstrate the feasibility and efficiency of using this bundle pricing approach. Our linear formulation solves the problem to optimality for large bundle sizes (up to 10 customer segments and 500 bundle sizes) in seconds. The exact solution is obtained using Gurobi optimization solver running on AMPL. Many instance of the problem are solved to optimality within fractions of a second, including instances with fairly high number of products.
This research adds to the literature of bundle pricing and guides multiproduct firms on how to optimally offer bundles under a practical and simple price structure. We show the gains of customized bundling over pure bundling in terms of increased profits.

Our approach assumes low marginal cost products. For future work, it might be worth investigating how the results change if this assumption is relaxed. It might also be useful to expand our generic model to more specific industries.

\section{REFERENCES}

[1] W. Hanson and K. R. Martin, "Optimal bundle pricing," Management Science, vol. 36, no, 2, pp. 155-174, 1990.

[2] G. J. Stigler, "United states v. loew's Inc.: A note on block- booking," The Supreme Court Review, 1963, pp. 152-157, 1963.

[3] W. J. Adams and J. L. Yellen, "Commodity bundling and the burden of monopoly," The Quarterly Journal of Economics, vol. 90, no. 3, pp. 475-498, 1976.

[4] R. Schmalensee, "Gaussian demand and commodity bundling," The Journal of Business, vol. 57, no. 1, pp. S211-S230, 1984.

[5] Y. Bakos and E. Brynjolfsson, "Bundling information goods: Pricing, profits, and efficiency," Management Science, vol. 45, no. 12, pp. 1613-1630, 1999.

[6] B. Yang, C. Ng, "Pricing problem in wireless telecommunication product and service bundling," European Journal of Operational Research, vol. 207, no. 1, pp. 473-480, 2010.

[7] S. Wu, L. M. Hitt, P. Chen, and G. Anandalingam, "Customized bundle pricing for information goods: A nonlinear mixed-integer programming approach," Management Science, vol. 54, no. 3, pp. 608-622, 2008

[8] L. M. Hitt, P. Chen, "Bundling with customer self-selection: A simple approach to bundling low-marginal-cost goods," Management Science, vol. 51, no. 10, pp. 1481-1493, 2005.

[9] C. S. Chu, P. Leslie, and A. Sorensen, "Bundle-size pricing as an approximation to mixed bundling," American Economic Review, vol. 101, no. 1, pp. 263-303, 2011.

[10] J. F. Wu, M. Tawarmalani, and K. N. Kannan, "Cardinality bundling with spence-mirrlees reservation prices," Management Science, vol. 65, no. 4, pp.1455-1947, 2018.

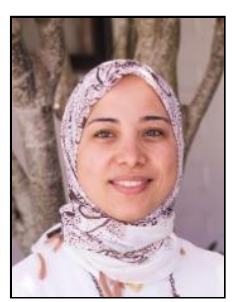

Ameera Ibrahim received her Ph.D. in management science and optimization from the University of Massachusetts Amherst with focus on Prescriptive Analytics and Mixed Integer Programming. She earned her M.S. in Computer Science from the University of Nantes in France, and her B.S. in Computer Science from Ain Shams University in Egypt.

She is currently an assistant professor of Business Analytics at the School of Economics and Business Administration, Saint Mary's College of California. Her current research interests include retail analytics, healthcare analytics and optimization-based heuristic approaches. 